\title{
Is neutrophilia associated with mortality of COVID-19? A meta-analysis and meta-regression
}

\author{
Li Shi $^{1}$, Ying Wang ${ }^{1}$, Xuan Liang ${ }^{1}$, Wenwei Xiao ${ }^{1}$, Guangcai Duan ${ }^{1}$, Haiyan Yang ${ }^{1}$, and \\ Yadong Wang ${ }^{2}$ \\ ${ }^{1}$ Zhengzhou University \\ ${ }^{2}$ Henan Province Center for Disease Control and Prevention
}

June 2, 2020

Is neutrophilia associated with mortality of COVID-19? A meta-analysis and meta-regression

Li Shi $^{1}$, Ying Wang ${ }^{1}$, Xuan Liang $^{1}$, Wenwei Xiao ${ }^{1}$, Guangcai Duan ${ }^{1}$, Haiyan Yang ${ }^{1 *}$, Yadong Wang ${ }^{2 *}$

${ }^{1}$ Department of Epidemiology, School of Public Health, Zhengzhou University, Zhengzhou 450001, China.

${ }^{2}$ Department of Toxicology, Henan Center for Disease Control and Prevention, Zhengzhou 450016, China.

*Corresponding author: Haiyan Yang, Department of Epidemiology, School of Public Health, Zhengzhou University, No. 100 of Science Avenue, Zhengzhou 450001, China. Phone: 86-371-67781248; Fax: 86371-67781248; Email address:yhy@zzu.edu.cn. Yadong Wang, Department of Toxicology, Henan Center for Disease Control and Prevention, No. 105 of South Nongye Road, Zhengzhou 450016, China. Email address: wangyd76@163.com.

Acknowledgments: This study was supported by a grant from the National Natural Science Foundation of China (No. 81973105).

Conflicts of interest: All authors report that they have no potential conflicts of interest.

Running title: Neutrophilia and mortality in COVID-19 patients

Keywords:COVID-19, Neutrophil, Mortality, Meta-analysis

Author contributions: Conceptualization: Li Shi, Haiyan Yang, Yadong Wang; Data extraction: Li Shi, Ying Wang, Xuan Liang, and Wenwei Xiao; Data analysis: Li Shi and Ying Wang; Methodology: Li Shi, Ying Wang, Guangcai Duan, Haiyan Yang, and Yadong Wang; Software: Li Shi, Xuan Liang, and Wenwei Xiao; Writing and review: Li Shi, Ying Wang, Haiyan Yang, Yadong Wang. All the authors approved the final manuscript.

\section{To the Editor,}

Coronavirus disease 2019 (COVID-19) is spreading rapidly around the world. There are plenty of emerging researches on the risk factors of severe and mortal COVID-19 patients. Huang et al reported that the elevated leukocyte counts and decreased lymphocyte counts were significantly associated with the severity of COVID19. Although neutrophil counts were not uniformly reported in that study, they thought that neutrophilia was more specific to severe patients than leukocytosis ${ }^{1}$. To our knowledge, a number of studies have investigated the association of neutrophil counts with the mortality of COVID-19, however, the conclusions among studies are contradictive ${ }^{2-6}$. On this basis, we explored the relationship between neutrophil counts and mortality of COVID-19 by quantitative meta-analysis and meta-regression. 
We completed our meta-analysis by strictly following the Preferred Reporting Items for Systematic Reviews and Meta-Analyses (PRISMA) guidelines. We conducted an electronic search of PubMed, Web of Science and Chinese National Knowledge Infrastructure to identify potential studies published between January 1, 2020 and May 22, 2020 using the following terms: ("clinical" OR "laboratory" OR "neutrophil") AND ("coronavirus" OR "2019-nCoV" OR "SARS-CoV-2" OR "COVID-19") AND ("outcome" OR "mortality"). In addition, the references of eligible studies were also reviewed by two researchers (Li Shi and Ying Wang), respectively. Extracted data included: authors, locations, number of cases per study, percentages of male, the median or mean of age, and neutrophil counts and corresponding units in the non-survival and survival groups.

The inclusion criteria involved (1) studies presented in English; (2) patients with laboratory-confirmed and clinically-diagnosed COVID-19 pneumonia; (3) clear report about neutrophil counts in the non-survival and survival groups. Case reports, meta-analysis, review, and studies with overlapping data were excluded.

Considering the inherent differences among studies, we calculated the pooled standardized mean difference (SMD) and corresponding 95\% confidence interval (CI) for continuous variables by using random-effects model to evaluate the relationship between changes in neutrophil counts and mortality of COVID-19 patients. When the mean and standard deviation could not be extracted directly from studies, we estimated them according to Wan et al's ${ }^{7}$ method by utilizing sample size, median and interquartile range (IQR) or median and range. The $I^{2}$ statistic and Cochran's Q statistic were used to quantify the heterogeneity across studies. For the Cochran's Q statistic, significant heterogeneity across studies was deemed as a $P$ value $<0.10$. For the $I^{2}$ statistic, significant heterogeneity across studies was regarded as $I^{2}>50 \%$. We used age and gender as covariates to conduct a restricted-maximum likelihood random effects meta-regression. Sensitivity analysis was used not only to identify sources of heterogeneity but also to assess the robustness of the results. For assessing small-study effects, we chose Begg's test and regression-based Egger's test. All calculations were performed in STATA 16.0 (StataCorp, College Station, TX, USA). Two-tailed $P$ values $<0.05$ were considered statistically significant.

At the beginning, there were 648 records in the search results, 100 duplicates were deleted, and the remaining 548 studies were screened. Finally, a total of 10 studies $^{2-6,8-12}$ having 1,473 COVID-19 cases were included through careful screening of titles, abstracts and full texts (Table 1).

The combined results revealed that higher neutrophil counts were detected in the non-survival COVID-19 patients compared with the survival COVID-19 patients $\left(\mathrm{SMD}=0.93,95 \% \mathrm{CI}=0.63-1.24, I^{2}=76.3 \%\right.$, Q $=42.12, P<0.001)$ (Fig. 1A). The results of sensitivity analysis suggested that removing any individual study of the included studies had no significant effect on the association between changes in neutrophil counts and mortality of COVID-19 infected patients (Fig. 1B). Due to the limitations of the data reported in the included studies, we only used age and gender as covariates for meta-regression. The results of meta-regression analysis indicated that the relationship between changes in neutrophil counts and increased risk of mortality in COVID-19 infected patients was not obviously affected by age $(P=0.628)$ (Fig. 1C) and gender $(P=0.222)$ (Fig. 1D). Begg's test $(P=1.839)$ and regression-based Egger's test $(P=0.058)$ demonstrated no small-study effects for the relationship between neutrophil counts and increased risk of mortality in COVID-19 patients.

Cytokine storms, characterized by aberrant activation of immune cells and excessive release of inflammatory cytokines (e.g., IL1 $\beta$, IFN $\gamma$, MCP1, and so on), were common in patients with severe COVID-19 infection, leading to severe lung injury, acute respiratory distress syndrome (ARDS), and even death ${ }^{13,14}$. Neutrophils are important immune cells and the first line of defense for the body's immune barrier, effecting against foreign invaders ${ }^{15}$. Neutrophils participate in the immune response to pathogen infection by phagocytosis and degranulation. Furthermore, a new mechanism through which neutrophils could kill pathogens reported by Brinkmann et al in $2004^{16}$. Neutrophils, stimulated by phorbol myristate acetate, lipopolysaccharide or interleukin 8, undergo programmed death and then eventually release neutrophil extracellular traps (NETs), a process called NETosis ${ }^{16}$. NETosis is a double-edged sword. Although NETs can protect the host from pathogens, excessive NET formation can also cause a hyperinflammatory condition, which lead to organ 
damage, including lung, kidney, and so on ${ }^{17,18}$. Multiple organ injuries are common in severe and fatal COVID-19 infected patients ${ }^{3,9}$. The aberrant activation of neutrophil-the ability to form excessive NETswas considered to be the core of the dysregulated host response ${ }^{19}$. Our current study demonstrated that the elevated neutrophil counts was significantly correlated to the mortality of COVID-19 patients. However, the meta-analysis was based on ten published studies with 1,473 COVID-19 cases. Therefore, future studies with larger sample size and no repeated data are needed to support our results. In conclusion, neutrophilia is a risk factor for mortality of COVID-19 patients, and treatment for neutrophilia to reduce the clinical severity of COVID-19 is worth considering, for example targeting NETs if appropriate.

\section{References}

1. Huang G, Kovalic AJ, Graber CJ. Prognostic Value of Leukocytosis and Lymphopenia for Coronavirus Disease Severity. Emerg Infect Dis. 2020;26(8). doi: 10.3201/eid2608.201160

2. Chen T, Dai Z, Mo P, et al. Clinical characteristics and outcomes of older patients with coronavirus disease 2019 (COVID-19) in Wuhan, China (2019): a single-centered, retrospective study. J Gerontol A Biol Sci Med Sci. 2020. doi: 10.1093/gerona/glaa089

3. Chen $\mathrm{T}$, Wu D, Chen $\mathrm{H}$, et al. Clinical characteristics of 113 deceased patients with coronavirus disease 2019: retrospective study. BMJ. 2020;368:m1091. doi: 10.1136/bmj.m1091

4. Du RH, Liang LR, Yang CQ, et al. Predictors of mortality for patients with COVID-19 pneumonia caused by SARS-CoV-2: a prospective cohort study. Eur Respir J. 2020;55(5). doi: 10.1183/13993003.00524-2020

5. Wu C, Chen X, Cai Y, et al. Risk Factors Associated With Acute Respiratory Distress Syndrome and Death in Patients With Coronavirus Disease 2019 Pneumonia in Wuhan, China. JAMA Intern Med. 2020. doi: 10.1001/jamainternmed.2020.0994

6. Martin-Moro F, Marquet J, Piris M, et al. Survival study of hospitalized patients with concurrent Covid-19 and haematological malignancies. Br J Haematol. 2020. doi: 10.1111/bjh.16801

7. Wan X, Wang W, Liu J, Tong T. Estimating the sample mean and standard deviation from the sample size, median, range and/or interquartile range. BMC Med Res Methodol. 2014;14:135. doi: 10.1186/1471$2288-14-135$

8. He W, Chen L, Chen L, et al. COVID-19 in persons with haematological cancers. Leukemia. 2020. doi: $10.1038 / \mathrm{s} 41375-020-0836-7$

9. Wang D, Yin $\mathrm{Y}, \mathrm{Hu} \mathrm{C}$, et al. Clinical course and outcome of 107 patients infected with the novel coronavirus, SARS-CoV-2, discharged from two hospitals in Wuhan, China. Crit Care. 2020;24(1):188. doi: $10.1186 / \mathrm{s} 13054-020-02895-6$

10. Wang K, Zuo P, Liu Y, et al. Clinical and laboratory predictors of in-hospital mortality in patients with COVID-19: a cohort study in Wuhan, China. Clin Infect Dis. 2020. doi: 10.1093/cid/ciaa538

11. Wang L, He W, Yu X, et al. Coronavirus disease 2019 in elderly patients: Characteristics and prognostic factors based on 4-week follow-up. J Infect. 2020;80(6):639-645. doi: 10.1016/j.jinf.2020.03.019

12. Yan Y, Yang Y, Wang F, et al. Clinical characteristics and outcomes of patients with severe covid-19 with diabetes. BMJ Open Diabetes Res Care. 2020;8(1). doi: 10.1136/bmjdrc-2020-001343

13. Huang C, Wang Y, Li X, et al. Clinical features of patients infected with 2019 novel coronavirus in Wuhan, China. Lancet (London, England). 2020;395(10223):497-506. doi: 10.1016/S0140-6736(20)30183-5

14. Channappanavar R, Perlman S. Pathogenic human coronavirus infections: causes and consequences of cytokine storm and immunopathology. Semin Immunopathol. 2017;39(5):529-539. doi: 10.1007/s00281-017$0629-\mathrm{x}$ 
15. Nauseef WM, Borregaard N. Neutrophils at work. Nat Immunol. 2014;15(7):602-611. doi: $10.1038 /$ ni. 2921

16. Brinkmann V, Reichard U, Goosmann C, et al. Neutrophil extracellular traps kill bacteria. Science (New York, NY). 2004;303(5663):1532-1535. doi: 10.1126/science.1092385

17. Liang X, Liu L, Wang Y, et al. Autophagy-driven NETosis is a double-edged sword - Review. Biomed Pharmacother. 2020;126:110065. doi: 10.1016/j.biopha.2020.110065

18. Papayannopoulos V. Neutrophil extracellular traps in immunity and disease. Nat Rev Immunol. 2018;18(2):134-147. doi: 10.1038/nri.2017.105

19. Barnes BJ, Adrover JM, Baxter-Stoltzfus A, et al. Targeting potential drivers of COVID-19: Neutrophil extracellular traps. The Journal of experimental medicine. 2020;217(6). doi: 10.1084/jem.20200652

Table 1. Characteristics of the included studies

\begin{tabular}{lllllllll}
\hline Author & Location & Case & Male (\%) & Age, years & Non-survival & Non-survival & Survival & S \\
\hline & & & & & $\mathrm{n}$ & Neutrophils, $\times 10^{9} / \mathrm{L}$ & $\mathrm{n}$ & $\mathrm{N}$ \\
Chen T et al. & China & 274 & $171(62.4)$ & 62 (median) & 113 & $9.0(5.4-12.7)$ & 161 & 3 \\
Chen Tielong et al. & China & 55 & $34(61.8)$ & 74 (median) & 19 & $5.5(2-21)$ & 36 & 4 \\
Du R et al. & China & 179 & $97(54.2)$ & $57.6($ mean) & 21 & $7.7(3.0-11.5)$ & 158 & 3 \\
He W et al. & China & 13 & $7(53.8)$ & 35 (median) & 8 & $0.7(0.2-6.5)$ & 5 & 2 \\
Wang D et al. & China & 107 & $57(53.3)$ & 51 (median) & 19 & $5.4(3.2-8.5)$ & 88 & 2 \\
Wang K et al. & China & 296 & $140(47.3)$ & $47.32($ mean) & 19 & $6.4(3.2-10.0)$ & 277 & 3 \\
& & 44 & $24(54.5)$ & $55.2($ mean) & 14 & $5.8(5.0-8.4)$ & 30 & 3 \\
Wang L et al. & China & 339 & $166(49.0)$ & $69($ median) & 65 & $7.65(4.35-11.74)$ & 274 & 4 \\
Wu C et al. & China & 84 & $60(71.4)$ & 58.5 (median) & 44 & $7.43(5.15-10.60)^{+}$ & 40 & 5 \\
Yan Y et al. & China & 48 & $33(68.8)$ & 69.4 (mean) & 39 & $8.04(5.36-12.49)$ & 9 & 3 \\
Martín-Moro F et al. & Spain & 34 & $19(55.9)$ & 72.5 (median) & 11 & $7.4(0-64.2)^{++}$ & 23 & 4 \\
\hline
\end{tabular}

All values are $\mathrm{n}(\%)$, or median (IQR). ${ }^{+}$, the unit of neutrophils is $\times 10^{9} / \mathrm{mL} ;{ }^{++}$, the values are median (range).

\section{Figure legend}

Fig.1. The pooled standardized mean difference (SMD) and corresponding 95\% confidence interval (CI) (A), sensitivity analysis (B), meta-regression for age (C) and gender (D) to evaluating the association between changes in neutrophil counts and mortality of COVID-19 infected patients. 
A
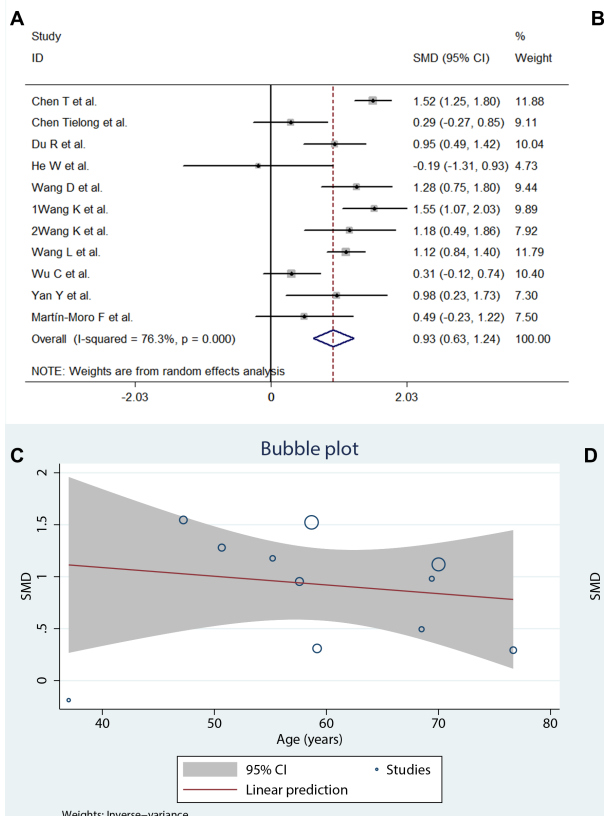
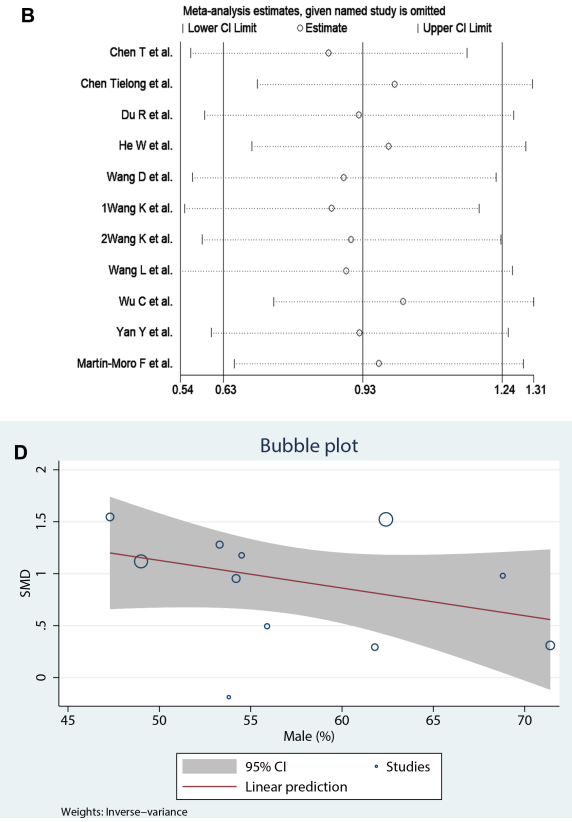